\title{
Monitorización neurofisiológica intraoperatoria en cirugía de columna
}

\section{Intraoperative neurophysiological monitoring in spine surgery}

\author{
L. Imirizaldu ${ }^{1}$, J. Urriza ${ }^{1}$, O. Olaziregi ${ }^{1}$, A. Hidalgo ${ }^{2}$, R.M. Pabón ${ }^{1}$
}

\section{RESUMEN}

La monitorización neurofisiológica intraoperatoria (MNIO) permite conocer el estado de las funciones neurológicas durante la cirugía, guiando al cirujano y minimizando los riesgos de lesión.

En este trabajo se describen las diferentes técnicas neurofisiológicas disponibles para la $\mathrm{MNIO}$ en cirugía del raquis (potenciales evocados somatosensoriales, potenciales evocados motores, neurografía, electromiografía, reflejos y potenciales evocados dermatómicos), que se emplearán a criterio del neurofisiólogo según las estructuras nerviosas en riesgo.

Para el éxito de la monitorización es indispensable la coordinación entre todos los profesionales del equipo. En el caso de que la MNIO muestre alteraciones, en primer lugar, el neurofisiólogo debe asegurar la integridad del sistema de registro. A continuación, el anestesista valorará la presión sanguínea, oxigenación, ventilación y hematocrito adecuados y revertirá los cambios recientes realizados en la anestesia. Por último, el cirujano, debería detener la intervención e intentar determinar la causa para revertirla en el caso de que fuera posible.

Palabras clave. Cirugía del raquis. Monitorización neurofisiológica intraoperatoria. Potenciales evocados motores. Potenciales evocados somatosensoriales. Estimulación transcraneal.

\begin{abstract}
Intraoperative neurophysiological monitoring (IONM) makes it possible to determine the status of neurological function during surgery. It guides the surgeon and minimises the risk of injury.

This paper describes the different techniques available for IONM in spine surgery (somatosensory evoked potentials, motor evoked potentials, neurography, electromyography, reflexes and dermatomic evoked potentials), which neurophysiologists employ depending on the nerve structures at risk.

In order for monitoring to be successful, coordination between all members of the surgical team is essential. In the event of IONM registering alterations, the neurophysiologist must first check the integrity of the recording system. The anaesthetist should then assess blood pressure, oxygen levels, ventilation and haematocrit values, and revert recent anaesthetic changes. Finally, the surgeon must stop the procedure and try to determine the cause of the event, and correct it if possible.
\end{abstract}

Key words. Spine surgery. Neurophysiological intraoperative monitoring. Motor evoked potentials. Somatosensory evoked potentials. Transcranial stimulation.
1. Servicio de Neurofisiología Clínica. Hospital Virgen del Camino. Pamplona.

2. Servicio de Traumatología y Cirugía

Ortopédica. Clínica Ubarmin. Elcano. Navarra.

\section{Correspondencia}

Lorea Imirizaldu Monente

Servicio de Neurofisiología Clínica

Hospital Virgen del Camino

Irunlarrea, 4

31008 Pamplona

Tfno. 848429474

E-mail: lorea.imirizaldu.monente@cfnavarra.es 


\section{INTRODUCCIÓN}

Las técnicas quirúrgicas empleadas en el tratamiento de las deformidades y estabilizaciones e inmovilizaciones de columna llevan implícito un cierto riesgo de lesión neurológica. La Scoliosis Research Society ha puesto de manifiesto que la incidencia de déficit neurológico en todas las formas de cirugía de columna es del $1,6 \%^{1}, \mathrm{y}$ que la incidencia específicamente en la cirugía de escoliosis es del $1,2 \%{ }^{2}$. El riesgo aumenta si existe un déficit neurológico previo.

Hasta la aparición de los potenciales evocados, los únicos medios de valoración del sistema nervioso de los que se disponía eran la corrección preoperatoria mediante tracción vertebral para determinar cuánta tracción se puede ejercer sin daño neurológico y el "test de despertar" intraoperatorio para valorar una posible lesión motora a grosso modo una vez terminada la corrección y antes de terminar la cirugía ${ }^{3}$.

Con el desarrollo de la monitorización neurofisiológica intraoperatoria (MNIO) de los potenciales evocados sensitivo-motores y de la electromiografía, se ha conseguido conocer el estado de las funciones neurológicas durante la cirugía en tiempo real. De esta forma, se intenta guiar al cirujano y minimizar los riesgos de lesión, proporcionando al paciente la mejor asistencia.

A pesar de que es difícil cuantificar el coste real de una monitorización, hay grandes evidencias de que el valor real de la misma no excede al de las secuelas, sobre todo, teniendo en cuenta el enorme costo de los cuidados y el sufrimiento humano relacionados con la lesión severa de la médula espinal ${ }^{4}$. Según un estudio realizado en el año 2004, la MNIO saldría rentable si se pudiera prevenir la paraplejia en una de cada 833 intervenciones ${ }^{5}$.

\section{RIESGOS QUIRÚRGICOS}

Las maniobras quirúrgicas potencialmente lesivas sobre la médula y las raíces son esencialmente de tres tipos:
1. Manipulación con implantes de osteosíntesis e instrumental de aplicación (que pueden producir lesión dural, parálisis radicular transitoria, dolor radicular, etc. $)^{6}$.

2. Cirugía de corrección de las deformidades con dos mecanismos posibles de lesión:

- Excesiva corrección de la curvatura de la columna vertebral, con el consiguiente estiramiento excesivo de haces nerviosos.

- Hipotensión y pérdida sanguínea prolongadas, con la consiguiente isquemia medular.

3. Técnicas de descompresión, fundamentalmente medulares, en pacientes con tumoraciones o fenómenos compresivos.

\section{TÉCNICAS NEUROFISIOLÓGICAS}

El objetivo de la MINO consiste en registrar continuamente la actividad eléctrica nerviosa provocada o espontánea, de forma que se puedan detectar los cambios que preceden a la lesión neurológica, de cara a evitar ésta. Para la MNIO en cirugía del raquis se utilizan varias técnicas: potenciales evocados somatosensoriales (PESS), potenciales evocados motores (PEM), electromiografía (EMG), electroneurografía (ENG), reflejos y potenciales evocados dermatómicos.

\section{POTENCIALES EVOCADOS SOMATOSENSORIALES}

Los PESS dan información sobre la integridad de las vías sensitivas (nervio periférico, cordones posteriores de la médula espinal y corteza cerebral sensitiva) $)^{7,8}$. Para su obtención se estimula un nervio periférico y se registran las respuestas en diferentes puntos a lo largo de la vía. Tanto la elección del nervio y el lugar a estimular como la de los lugares de registro vienen determinadas por el nivel de la cirugía.

Estas respuestas evocadas son muy pequeñas, por lo que es necesario sumarlas mediante técnicas de promediado para extraerlas de la actividad electroencefalo- 
gráfica y del ruido de fondo. Esto impide la detección inmediata de los cambios producidos por las maniobras quirúrgicas, por lo que para intentar maximizar la sensibilidad de esta técnica se debe realizar a intervalos frecuentes.

Los registros periféricos (hueco poplíteo, fosa supraclavicular) permiten valorar el correcto funcionamiento del sistema de registro y/o los efectos sistémicos de la cirugía o de la anestesia en el paciente (hipotensión, hipovolemia, fármacos, etc.).

Habitualmente se aceptan como señales de alarma tanto la disminución de la amplitud mayor del 50\% como el aumento de la latencia mayor del $10 \%$ respecto a las respuestas basales. La pérdida transitoria de los potenciales con posterior recuperación indica una lesión medular posible y la pérdida completa y persistente, una lesión medular segura, al menos de los cordones posteriores. Éstos tienen una irrigación diferente a la parte anterior de la médula, por lo que su normalidad no necesariamente es indicativa de indemnidad de las vías motoras o de las astas anteriores ${ }^{9}$.

Los PESS no siempre se afectan en una lesión radicular aislada, ya que los troncos nerviosos se componen de más de una raíz y por ello las fibras intactas de las otras raíces pueden enmascarar dicha lesión.

\section{POTENCIALES EVOCADOS MOTORES}

Los PEM dan información sobre la integridad de las vías motoras, ya que mediante la estimulación eléctrica transcraneal se puede producir la despolarización de las neuronas corticoespinales y los impulsos descendentes se pueden registrar tanto en la médula espinal como en los músculos de las extremidades.

El registro realizado en la médula muestra una onda $\mathrm{D}$ (por activación directa de las neuronas corticoespinales) y puede mostrar una o varias ondas I (por activación transináptica ${ }^{10}$. En el músculo se registra un potencial de acción muscular compuesto polifásico y de morfología variable de un estímulo a otro.
La onda $\mathrm{D}$ es un registro directo de la función del haz corticoespinal y es relativamente insensible a la anestesia. Sin embargo, para su obtención es imprescindible la introducción de electrodos epidurales y cierto grado de relajación muscular. Además, no identifica el lado de la afectación y no se puede obtener a niveles lumbares ${ }^{7}$. Se han descrito cambios en la onda D en cirugías de escoliosis que no se han seguido de cambios clínicos, lo que demuestra sus posibles falsos positivos-negativos en este tipo de intervenciones ${ }^{11}$.

Como ya queda dicho, uno de los mecanismos de lesión más importante en la cirugía de columna es la isquemia espinal, que puede provocar la pérdida de los PEM en unos 2 minutos. Aún así, tenemos un cierto margen de actuación, ya que el infarto no comienza hasta más o menos los 10 minu$\operatorname{tos}^{12}$.

En el caso del registro de la onda D, el criterio de alarma es la disminución de la amplitud del potencial de más del $50 \%{ }^{13}$ y en el caso del registro muscular, el aumento brusco del umbral de estimulación mayor del $50 \%{ }^{14}$ o la desaparición de la respuesta.

La estimulación eléctrica transcraneal no está exenta de efectos secundarios, entre los que se encuentran la laceración lingual-labial, la fractura mandibular, las crisis epilépticas, las arritmias cardiacas o las quemaduras de scalp, entre otros ${ }^{5}$. Se consideran contraindicaciones absolutas la hipertensión intracraneal, las válvulas o los marcapasos cardíacos y contraindicación relativa la epilepsia ${ }^{8}$.

\section{ELECTROMIOGRAFÍA}

La EMG es la técnica que permite visualizar y escuchar durante todo el procedimiento quirúrgico la actividad eléctrica muscular espontánea que se puede producir en los músculos inervados por las raíces consideradas en riesgo de lesión intraoperatoria debida a la posible irritación de las mismas ${ }^{7,15-17}$ (Tabla 1). Aunque no predice necesariamente el resultado posquirúrgico, minimiza la posibilidad de radiculopatía secundaria a cirugía de columna. 
Tabla 1. Músculos guía adecuados para la MNIO.

\begin{tabular}{cl}
$\begin{array}{c}\text { Raíces en } \\
\text { riesgo }\end{array}$ & \multicolumn{1}{c}{\begin{tabular}{c}
\multicolumn{1}{c}{ Músculos adecuados } \\
para MNIO de EMG
\end{tabular}} \\
\hline C3-C4 & Trapecio \\
C5-C6 & Bíceps \\
C6-C7 & Tríceps \\
C7-C8 & Extensor común de los dedos \\
T1 & Separador corto del pulgar \\
T2-T6 & Intercostales \\
T7-T12 & Oblicuo externo y recto abdominal \\
L1-L2 & Psoas-iliaco \\
L2-L4 & Vasto medial \\
L4-L5 & Tibial anterior \\
S1-S2 & Gemelo medial \\
S3-S5 & Esfínteres anal y uretral \\
\hline
\end{tabular}

Las maniobras que implican irritación de la raíz, como isquemia, manipulación, irrigación, compresión o tracción de las mismas, pueden generar series de potenciales de unidad motora irregulares de hasta varios segundos de duración (descargas neurotónicas) (Fig. 1) en los músculos inervados por ellas, por lo que el cirujano debe detener toda manipulación y esperar a la desaparición de estas descargas. Si no desaparecen, se deberá investigar la causa y solucionarla siempre que sea posible. No obstante, hay que tener en cuenta que la sección total del nervio puede también no producir ningún potencial.

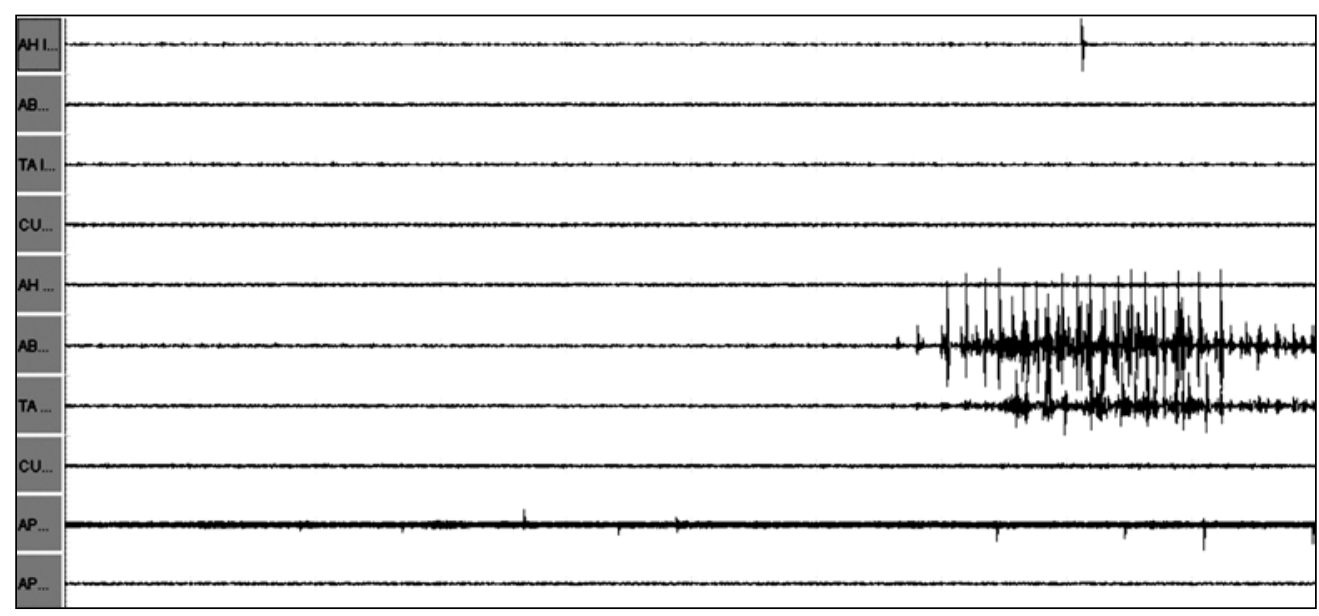

Figura 1. Descarga neurotónica.

Cuando el músculo ya está parcialmente denervado se puede registrar actividad espontánea presente previamente (fibrilaciones, mioquimias, descargas repetitivas complejas, etc.) y en este caso es posible que la manipulación de la raíz provoque menos descargas neurotónicas, por lo que pueden no advertirse nuevas lesiones en dicha raíz.

Las descargas neurotónicas deben diferenciarse de otros tipos de actividades (artefactos) que se pueden llegar a registrar:

- Patrón de "repiqueteo»: puede ocurrir durante la exposición, exploración, descompresión, artrodesis e instrumentación, y está causado por el impacto de los instrumentos quirúrgicos o el injerto contra los elementos óseos de la columna.

- Patrón electromiográfico de «despertar»: se observa cuando aumenta el 
tono muscular debido a que la profundidad de la anestesia es insuficiente para prevenir el movimiento en un paciente sin relajación muscular. Cuando aparece este patrón se debe avisar al anestesista y al cirujano de la posibilidad de movimiento y se debe detener cualquier estímulo doloroso.

\section{ELECTRONEUROGRAFÍA}

La ENG permite localizar estructuras nerviosas y valorar su integridad, aplicando estímulos eléctricos directa o indirectamente en el tejido nervioso y registrando las respuestas evocadas sobre los músculos de su correspondiente miotomo $0^{7,8,15,16,18}$.

En la cirugía de columna, esta técnica tiene dos aplicaciones principales: la identificación de estructuras neurales y la valoración de la idoneidad de la colocación de los tornillos pediculares con respecto a las estructuras neurales cercanas. En este último caso, el fundamento electrofisiológico en el que nos apoyamos lo constituye el hecho de que la intensidad de estímulo necesaria para conseguir una respuesta variará en función de la cantidad de tejido que se encuentre entre el tornillo estimulado y la raíz.

Así, la estimulación eléctrica se aplica en el lecho pedicular, en el palpador o en el propio tornillo ya insertado, de tal manera que si la pared ósea está intacta, el hueso actuará como una barrera al paso de la corriente eléctrica y se necesitará una intensidad de estimulación relativamente alta. Por el contrario, si hay alguna brecha en la pared, la intensidad necesaria para producir una respuesta será menor.

Los límites de los umbrales de estimulación varían en los distintos trabajos, pero se puede hablar de que una intensidad de estimulación menor de $6 \mathrm{~mA}$ sería indicativa de rotura de la pared pedicular y de contacto del tornillo con la raíz nerviosa, y de que intensidades entre $6-10 \mathrm{~mA}$ indicarían una posible rotura del pedículo, aunque sin contacto entre el tornillo y la raíz. Estos límites varían según la zona espinal en la que se instrumenta el tornillo, así como en casos de compresión crónica de la raíz, osteoporosis, etc. Se pueden observar episodios de descargas neurotónicas durante la manipulación de los palpadores o de los implantes pediculares, lo que puede estar indicando también rotura de la pared pedicular.

\section{REFLEJOS}

\section{Reflejo bulbocavernoso (RBC)}

La monitorización de este reflejo valora la integridad de los segmentos sacros S2-S4 y de sus aferencias y eferencias. Para ello, se estimula el nervio dorsal del pene o del clítoris y se registra la actividad eléctrica en el músculo esfínter anal externo. La presencia del RBC al final de la intervención indicaría la preservación de las funciones sacras en general ${ }^{15}$.

\section{Reflejo H}

Este reflejo segmentario monosináptico sería muy útil en la evaluación de la raíz sacra $\mathrm{S} 1$, pero debido a las dificultades en su registro se han publicado pocos trabajos relativos a su monitorización. Se ha utilizado en las rizotomías parciales selectivas ${ }^{15}$, durante la evacuación de discos herniados ${ }^{19}$ y como marcador del grado de retracción durante dichas cirugías ${ }^{20}$.

\section{POTENCIALES EVOCADOS DERMATÓMICOS}

Esta técnica valora la integridad funcional de las raíces sensitivas mediante la estimulación del dermatoma dependiente de la raíz en posible riesgo y el registro de las respuestas en la corteza somatosensorial.

Estos potenciales ayudan a detectar cualquier manipulación radicular durante la cirugía, pero tiene las mismas desventajas que los PESS (respuestas pequeñas, necesidad de promediado, no detección inmediata de lesión). Además, pueden no obtenerse en pacientes con historia previa de radiculopatía ${ }^{16}$. 


\section{TRABAJO MULTIDISCIPLINAR}

La MNIO necesita de una buena coordinación entre todos los profesionales del equipo y obliga a que se haga un trabajo multidisciplinar donde cada miembro sepa en cada momento cuál es su función.

El neurofisiólogo debe, por un lado, identificar la lesión nerviosa en el momento en que se produce, cuando todavía el cirujano puede tomar medidas para reducirla o revertirla, y por otro, definir la naturaleza de la misma, de modo que el cirujano pueda adaptar el procedimiento quirúrgico minimizando el riesgo de empeorar el daño. Para ello se utilizan las diferentes técnicas antes mencionadas, previamente seleccionadas según las estructuras nerviosas en riesgo (Tabla 2).

Tabla 2. Algunos protocolos utilizados en la técnica MNIO.

\begin{tabular}{ll}
\hline \multicolumn{1}{c}{ Cirugía } & \multicolumn{1}{c}{ Técnica de MNIO } \\
\hline Lumbar simple sin instrumentación & - No hay recomendaciones especiales \\
\hline Lumbar con instrumentación & - Neurografía y electromiografía. \\
& - En escoliosis degenerativa, osteotomía de columna lumbar o reduc- \\
& ción de espondilolistesis: añadir PESS y PEM. \\
& - Reflejo H. \\
\hline Torácica o toraco-abdominal & - PESS, PEM. \\
& - Neurografía y electromiografía. \\
\hline Cervical & - PESS, PEM. \\
& - Neurografía y electromiografía. \\
\hline Independientemente del tipo de cirugía & - PESS de EESS para valorar el plexo braquial. \\
& - Mapeo de raíces. \\
\hline
\end{tabular}

Para el éxito de la monitorización es indispensable la colaboración del anestesista, que deberá aplicar un protocolo anestésico que maximice la adquisición de las señales eléctricas.

La anestesia propuesta para la monitorización, según varios estudios, pasaría por la infusión continua de remifentanilo $(0,2-0,9 \mu \mathrm{g} / \mathrm{kg} / \mathrm{min})$ y propofol $(3-10 \mathrm{mg} /$ $\mathrm{kg} / \mathrm{h})$. Alternativa a esta pauta podría ser la ketamina (2-6 mg/kg/h). En la intubación debería utilizarse algún relajante de vida media muy corta de cara a poder registrar las líneas de base al inicio de la cirugía, pero no en otros tiempos del acto quirúrgico, donde se debería evitar la relajación, con el posible paréntesis temporal de la disección muscular de la espalda. Los gases anestésicos, especialmente los halogenados, como el sevoflurano (Fig. 2), no deberían utilizarse, y se halla bajo discusión si se puede usar o no el $\mathrm{N}_{2} \mathrm{O}$, aunque la mayoría de los autores abogan por no usarlo ${ }^{21}$.
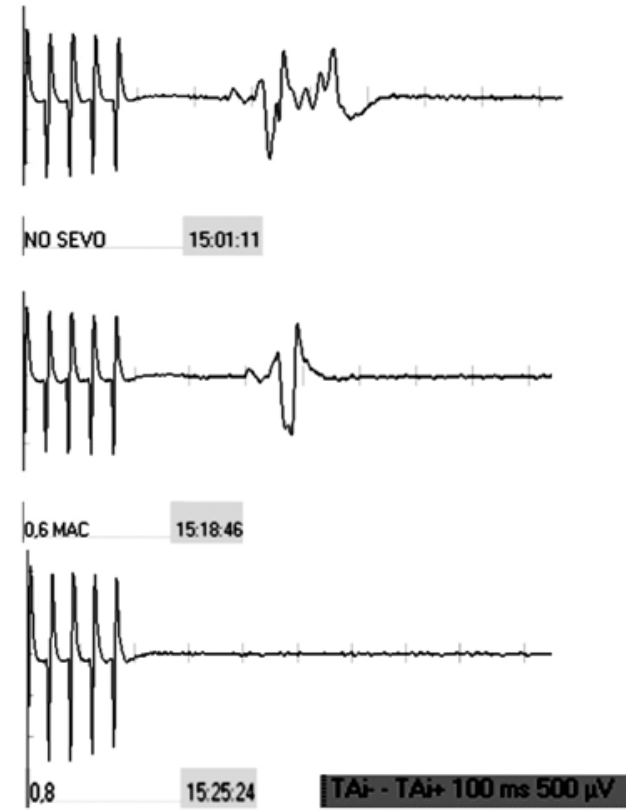

Figura 2. Efecto del sevoflurano en los PEM. 
De todas las modalidades de monitorización, los PEM son particularmente sensibles a los agentes anestésicos, todavía más si existe una afectación neurológica previa. La mayoría de estos fármacos deprimen la amplitud de las respuestas evocadas y aumentan la latencia, dificultando la MNIO. Sin embargo, los anestésicos intravenosos etomidato y ketamina son excepciones notables, ya que aumentan las amplitudes de las respuestas somatosensoriales y motoras (Tabla 3).

Tabla 3. Efectos de los distintos fármacos anestésicos sobre las técnicas neurofisiológicas habituales.

\begin{tabular}{|c|c|c|c|c|c|c|}
\hline \multicolumn{7}{|c|}{ Anestésicos } \\
\hline $\begin{array}{l}\text { Potenciales } \\
\text { evocados }\end{array}$ & Halogenados & Óxido nitroso & Propofol & Narcóticos & Ketamina & $\begin{array}{l}\text { Bloqueantes } \\
\text { unión } \\
\text { neuromuscular }\end{array}$ \\
\hline $\begin{array}{l}\text { PESS } \\
\text { corticales }\end{array}$ & $\begin{array}{l}\uparrow \text { latencia } \\
\downarrow \text { A. dosis } \\
\text { dependiente }\end{array}$ & $\begin{array}{l}\downarrow \text { A. } \\
\uparrow \text { latencia }\end{array}$ & & & $\uparrow \mathrm{A}$. & \\
\hline $\begin{array}{l}\text { PESS } \\
\text { subcorticales }\end{array}$ & & $\begin{array}{l}\text { Cambios } \\
\text { mínimos }\end{array}$ & & & & \\
\hline $\begin{array}{l}\text { PEM } \\
\text { estimulación } \\
\text { transcraneal }\end{array}$ & $\begin{array}{l}\downarrow \text { A. o } \\
\text { abolición }\end{array}$ & $\begin{array}{l}\downarrow \text { A. dosis } \\
\text { dependiente }\end{array}$ & $\begin{array}{l}\downarrow \text { A. con altas } \\
\text { dosis. } \\
\text { No efectos lat. }\end{array}$ & $\begin{array}{l}\text { Efectos } \\
\text { limitados }\end{array}$ & $\uparrow A$. & $\begin{array}{l}\downarrow \text { A. o } \\
\text { abolición }\end{array}$ \\
\hline EMG & & & & & & $\begin{array}{l}\downarrow \text { A. o } \\
\text { abolición }\end{array}$ \\
\hline
\end{tabular}

A: amplitud. $\uparrow:$ aumento. $\downarrow$ : disminución.

Independientemente del tipo de anestésico utilizado se debe mantener una concentración constante siempre que sea posible, debido a que alteraciones rápidas en la misma puede llevar a interpretar las alteraciones de los potenciales evocados como una lesión neuronal debida a la cirugía ${ }^{8,10}$.

La electromiografía no se puede monitorizar en presencia de bloqueo farmacológico de la placa neuromuscular, debido a que el impulso nervioso necesita su integridad funcional para evocar respuestas musculares identificables ${ }^{17}$.

El grado de bloqueo neuromuscular se puede valorar mediante dos técnicas:

1. La amplitud del potencial de acción muscular obtenido con estimulación supramaximal de un nervio motor, que no debe ser menor del $80 \%$ respecto a la respuesta basal.

2. El test de cuatro, que consiste en la aplicación de una serie de cuatro pulsos eléctricos supramaximales en un nervio motor y el registro de las respuestas: cero respuestas indican relajación profunda y 4 ausencia de relajación.

En el caso de que la MNIO mostrara alteraciones, todo el equipo quirúrgico debe trabajar unido para solucionar el problema. El neurofisiólogo debe asegurarse de la integridad del sistema de registro y el anestesista debe asegurar la presión sanguínea, oxigenación, ventilación y hematocrito adecuados y revertir los cambios recientes realizados en la anestesia. El cirujano, por su parte, debería detener por un momento la intervención hasta aclarar la naturaleza de los cambios registrados. Descartadas las causas técnicas y anestésicas, debe considerarse la cirugía como causante de las alteraciones.

En este caso, se intentará determinar si las modificaciones se deben a algún evento específico (en ocasiones difícil de valorar, debido a que la pérdida de poten- 
ciales se manifiesta de manera diferida al acto quirúrgico) y, si es así, revertir el evento supuestamente problemático. Si los potenciales evocados se recuperan se puede continuar con la cirugía, pero en caso contrario se debe revertir, si es posible, el gesto realizado antes de la alteración de la MNIO o, incluso, valorar una retirada del material de osteosíntesis; esto es fundamental en el caso de cirugía de corrección de las deformidades. Si a pesar de la retirada de la instrumentación los potenciales no vuelven a la normalidad se debe realizar un test de despertar ${ }^{8}$.

\section{CONSIDERACIONES FINALES}

La MNIO en la cirugía de la columna es sumamente útil en la corrección de deformidades espinales con escoliosis superiores a $45^{\circ}$, en la corrección de anomalías espinales congénitas, en la resección de tumores intra y extramedulares y en la descompresión extensa anterior y/o posterior en estenosis cervicales, torácicas o lumbares que causen mielopatía o alteración funcional de la cauda equina y/o raíces nerviosas ${ }^{21}$.

Existe un cierto consenso en que para una correcta monitorización sería necesaria la anestesia total intravenosa con infusión continua de remifentanilo y propofol, con posibilidad de usar un relajante de vida media muy corta en la intubación. Los gases anestésicos, especialmente los halogenados, no deberían utilizarse.

Para finalizar, no debemos olvidar que sería recomendable realizar una exploración neurológica y neurofisiológica previa a la intervención para conocer el estado funcional de las estructuras nerviosas. Asimismo, el neurofisiólogo debería conocer la técnica quirúrgica para distinguir los momentos de mayor riesgo y tener un conocimiento amplio de las técnicas neurofisiológicas para poder evaluar el posible daño neurológico y controlar los problemas técnicos y anestésicos que puedan surgir.

Por todo lo que hemos dicho en este artículo y las pruebas aportadas, creemos que todas las intervenciones con riesgo potencial de lesión de las diferentes estruc- turas nerviosas se deberían monitorizar de forma rutinaria. Esto conseguiría una disminución de la morbilidad periquirúrgica y un aumento de los indicadores de calidad del hospital ${ }^{16}$.

\section{BIBLIOGRAFÍA}

1. Dawson EG, Sherman JE, Kanim LE, Nuwer MR. Spinal cord monitoring. Results of the scoliosis research society and the european spinal deformity society survey. Spine 1991; 16 (Suppl. 8): 361-364.

2. Scoliosis research society. Scoliosis research society morbidity and mortality committee summary report for 2005. Scoliosis Research Society S 2006.

3. EgGspuehler A, Sutter MA, Grob D, Jeszenszky D, DVORAK J. Multimodal intraoperative monitoring during surgery of spinal deformities in 217 patients. Eur Spine J 2007; 16 (Suppl. 2): 188-196.

4. SAla F, DvoraK J, FAccioli F. Cost effectiveness of multimodal intraoperative monitoring during spine surgery. Eur Spine J 2007; 16 (Suppl. 2): 229-231.

5. Deletis V, Sala F. Intraoperative neurophysiological monitoring during spine surgery: an update. Curr Opin Orthop 2004; 15: 154-158.

6. Bose B, Wierzbowski LR, Sestokas AK. Neurophysiologic monitoring of spinal nerve root function during instrumented posterior lumbar spine surgery. Spine 2002; 27: 1444-1450.

7. Comisión de la sociedad española de neurofisiología clínica. Guía práctica para la realización de la monitorización neurofisiológica de la cirugía de columna. Rev Neurol 2004; 38: 879-885

8. Pajewsi TN, Arlet V, Phillips LH. Current aproach on spinal cord monitoring: the point of view of the neurologist, the anesthesiologis and the spine surgeon. Eur Spine J 2007; 16 (Suppl. 2): 115-129.

9. Lesser RP, Raudzens P, Lüders H, Nuwer MR, GoLDIE WD, MorRIS HH 3RD et al. Postoperative neurological deficits may occur despite unchanged intraoperative somatosensory evoked potentials. Ann Neurol 1986; 19: 22-25.

10. Deletis V, Isgum V, Amassian VE. Neurophysiological mechanisms underlying motor evoked potentials in anesthetized humans. Part 1. Recovery time of corticoespinal tract direct waves elicited by pairs of transcranial electrical stimuli. Clin Neurophysiol 2001; 112: 438-444. 
11. Ulkatan S, Neuwirth M, Bitan F, Minardi C, KoKoszka A, Deletis V. Monitoring of scoliosis surgery with epidurally recorded motor evoked potentials (D wave) revealed false results. Clin Neurophysiol 2006; 117: 2093-2101.

12. MacDonald DB, Zayed ZA, SAdDigi AA. Four-limb muscle motor evoked potencial monitoring with decussation assesment: result in 206 thoracolumbar spine surgeries. Eur Spine J 2007; 16 (Suppl. 2): 171-187.

13. Deletis V, Sala F. Intraoperative neurophysiological monitoring of the spinal cord during spinal cord and spine surgery: a review focus on the corticospinal tracts. Clin Neurophysiol 2008; 119: 248-264.

14. Calancie B, Harris W, Broton JG, Alexeeva N, GREEN BA. "Threshold-level» multipulse transcranial electrical stimulation of motor cortex for intraoperative monitoring of spinal motor tracts: description of method and comparison to somatosensory evoked potencial monitoring. J Neurosurg 1998; 88: 457-470.

15. KothBauER KF, NovaK K. Intraoperative monitoring for tethered cord surgery: an update. Neurosurg Focus 2004; 16: Article 8.
16. WEISS DS. Spinal cord and nerve root monitoring during surgical treatment of lumbar stenosis. Clin Orthop 2001; 384: 82-100.

17. HoLLAND NR. Intraoperative electromyography. J Clin Neurophysiol 2002; 19: 444-453.

18. Deletis V, Sala F. Basic methodological principles of multimodal intraoperative monitoring during spine surgeries. Eur J Spine 2007; 16 (Suppl. 2): 147-152.

19. Makovec M, Benedicic M, BosnjaK R. H wave and spinal root potentials in neuromonitoring of $\mathrm{S} 1$ root function during evacuation of herniated disc: preliminary results. Croat Med J 2006; 47: 298-304.

20. Logigian EL, Soriano SG, Herrmann DN, Madsen JR. Gentle dorsal root retraction and dissection can cause areflexia: implications for intraoperative monitoring during "selective" partial dorsal rhizotomy. Muscle Nerve 2001; 24: 1352-1358.

21. Sutter M, Deletis V, Dvorak J, Eggspuehler A, Grob D, MacDonald DB et al. Current opinions and recommendations on multimodal intraoperative monitoring during spine surgeries. Eur Spine J 2007; 16 (Suppl. 2): 232-237. 
